\title{
A NOTE ON GENERATING RELATIONS FOR LAURICELLA'S FUNCTION OF SEVERAL VARIABLES
}

\author{
D. P. SHUKLA
}

\begin{abstract}
In this paper we have derived generating relations for Lauricella's function by using the operator $T_{k}=x(k+x D)$ and the operational relations involving this operator. Some recent results of Srtvastava [3], Srivastava and Carlitz [4], Sharma and Abiodun [1] have been conveniently obtained by this method as well as some hitherto unknown results established.
\end{abstract}

1. Introduction. Srivastava [2], [3] considered the function

$$
f\left(z_{1}, \ldots, z_{r}\right)=\sum_{k_{1}, \ldots, k_{r}=0}^{\infty} C_{k_{1}, \ldots, k_{r}} \prod_{j=1}^{r} \frac{\left(z_{j}\right)^{k_{j}}}{k_{j} !}
$$

where $C_{k_{1}, \ldots, k_{r}}$ are arbitrary complex constants, and derived the generating relation (2.2). Considering the function in (1.1), Carlitz and Srivastava [4] obtained several generating functions for Lauricella's function of several variables.

Agarwal [8] and Sharma and Abiodun [1] considered the general Gfunction (4.1) and Sharma and Abiodun [1] derived a generating relation for it.

In this paper, making use of operational methods, we propose to derive results of Srivastava [3], Carlitz and Srivastava [4], Sharma and Abiodun [1] and other results directly by making use of the operator formulas (2.1), (3.1), (3.4), (3.6) and (3.8).

In $\$ 2$ we obtain a generating relation for $f\left(z_{1}, \ldots, z_{r}\right)$ defined in (1.1) and, in $\S 3$, we derive various generating functions for Lauricella's function of several variables. In $\$ 4$ we consider the general $G$-function and, using (2.1), we derive directly the result due to Sharma.

The series occurring in the paper may be thought of as formal power series.

2. Generating relations for functions of several variables. In what follows we shall make use of the following Mittal [7] operational generating formula

$$
\sum_{n=0}^{\infty} \frac{z^{n}}{n !} T_{a+1+(m-1) n}^{n} f(x)=\frac{(1+v)^{a+1}}{1-(m-1) v} f[x(1+v)]
$$

Received by the editors March 31, 1977.

AMS (MOS) subject classifications (1970). Primary 33A65.

Key words and phrases. Lauricella's functions of several variables, generating relations.

(c) 1979 American Mathematical Society 0002-9939/79/0000-0062/\$03.00 
where $v=x z(1+v)^{m}, m$ being constant and $f(x)$ admits a formal power series in $x$ and $T_{k} \equiv x(k+x D), D=d / d x$.

Assuming that the operator $T_{k}$ operates on $t$ alone and putting $a=\alpha$, $m=\beta+1$ and

$$
f(x) \equiv f\left[x_{1}\left(-y^{-\beta_{t} 1+\beta}\right)^{m_{1}}, \ldots, x_{r}\left(y^{1-\beta_{t} \beta}\right)^{m_{r}}\right] \text { in }(2.1)
$$

we immediately get

$$
\begin{aligned}
& \sum_{n=0}^{\infty}\left(\begin{array}{c}
\alpha+(\beta+1) n \\
n
\end{array}\right) t^{n_{2} n} \sum_{k_{1}, \ldots, k_{r}=0}^{n} \frac{(-n)_{\sum m_{i} k_{i}} z^{-\sum m_{i} k_{i}}}{(1+\alpha+\beta n)_{\sum m_{i} k_{i}}} \\
& \cdot C\left(k_{1}, \ldots, k_{r}\right) t^{\beta \Sigma_{m_{i} k_{j}}-\beta_{\Sigma m_{i k_{i}}}} \frac{x_{1}^{k_{1}}}{k_{1} !} \cdots \frac{x_{r}^{k_{r}}}{k_{r} !} \\
& =(1+v)^{\alpha+1}(1-\beta v)^{-1} f\left[x_{1}\left[-y^{-m_{1} \beta}\left\{t^{\beta+1}(1+v)^{\beta+1}\right\}^{m_{1}}\right], \ldots,\right. \\
& \left.x_{r}\left[-y^{-m_{r} \beta}\left\{t^{\beta+1}(1+v)^{\beta+1}\right\}^{m_{r}}\right]\right] \text {, }
\end{aligned}
$$

where $v=t z(1+v)^{\beta+1}$. Now putting $z=1$ and $y=t$ in (2.2) we get

$$
\begin{aligned}
\sum_{n=0}^{\infty}\left(\begin{array}{c}
\alpha+(\beta+1) n \\
n
\end{array}\right) t^{n} \sum_{k_{1}, \ldots, k_{r}=0}^{n} \frac{(-n)_{\sum m_{i} k_{i}}}{(1+\alpha+\beta n)_{\sum m_{i} k_{i}}} \\
\cdot C\left(k_{1}, \ldots, k_{r}\right) \frac{x_{1}^{k_{1}}}{k_{1} !} \cdots \frac{x_{r}^{k_{r}}}{k_{r} !} \\
=(1+v)^{\alpha+1}(1-\beta v)^{-1} f\left[x_{1}(-v)^{m_{1}}, \ldots, x_{r}(-v)^{m_{r}}\right]
\end{aligned}
$$

where $v=t(1+v)^{\beta+1}$, which is due to Srivastava [2].

3. Lauricella's function of several variables. In this section we shall use the Mittal [6] operational formulae

$$
\sum_{n=0}^{\infty} \frac{1}{n !} T_{a+1}^{n}\{f(x)\}=(1-x)^{-a-1} f\left[\frac{x}{1-x}\right]
$$

where $f(x)$ admits a formal power series in $x$. Putting $a=\lambda-1$, and

$$
f(x) \equiv F_{D}^{r}\left[\left(\lambda, m_{i}\right):\left(\beta_{i}, \varphi_{i}\right) ;\left(\gamma, \psi_{i}\right) ; x_{1}(-t)^{m_{1}}, \ldots, x_{r}(-t)^{m_{r}}\right]
$$

in (3.1) and assuming that $T_{k}$ operates on $t$ alone, we get

$$
\begin{aligned}
& \sum_{n=0}^{\infty} \frac{(\lambda)_{n}}{n !} F_{D}^{(r)}\left[\left(-n, m_{i}\right):\left(\beta_{i}, \varphi_{i}\right) ;\left(\gamma_{i}, \psi_{i}\right) ; x_{1}, \ldots, x_{r}\right] t^{n} \\
& =(1-t)^{-\lambda} F_{D}^{(r)}\left[\left(\lambda, m_{i}\right):\left(\beta_{i}, \varphi_{i}\right) ;\left(\gamma, \psi_{i}\right) ; x_{1}\left(\frac{t}{t-1}\right)^{m_{1}}, \ldots, x_{r}\left(\frac{t}{t-1}\right)^{m_{r}}\right],
\end{aligned}
$$


which is due to Carlitz and Srivastava [4]. Carlitz and Srvistava [4] also proved that

$$
\begin{aligned}
& \sum_{n=0}^{\infty} \frac{(\lambda)_{n}(\gamma)_{n} t^{n}}{n !(\mu)_{n}} F_{D}^{(r)}\left[\left(-n, m_{i}\right):\left(\beta_{i}, \varphi_{i}\right) ;\left(\gamma, m_{i}\right) ; x_{1}, \ldots, x_{r}\right] \\
&=(1-t)^{-\lambda} F_{D}^{(r+1)}\left[\left(\lambda, m_{i}, 1\right):\left(\beta_{i}, \varphi_{i}\right),(\mu-\gamma, 1) ;\left(\mu ; m_{i}, 1\right)\right. \\
&\left.x_{1}\left(\frac{t}{t-1}\right)^{m_{1}}, \ldots, x_{r}\left(\frac{t}{t-1}\right)^{m_{r}}, \frac{t}{t-1}\right] .
\end{aligned}
$$

Putting $a=\lambda-1$ and

$$
\begin{aligned}
f(x)=F_{D}^{(r+1)}\left[\left(\lambda, m_{i}, 1\right):\left(\beta_{i}, \varphi_{i}\right),\right. & (\mu-\gamma, 1) ;\left(\mu, m_{i}, 1\right) ; \\
& \left.x_{1}(-t)^{m_{1}}, \ldots, x_{r}(-t)^{m_{r}},(-t)\right]
\end{aligned}
$$

in (3.1) and assuming that $T_{k}$-operates on $t$ alone, and using Euler's transformation, the result in (3.3) follows. Again by making use of the Mittal [6] operational formula

$$
\begin{aligned}
\sum_{n=0}^{\infty} \frac{1}{n !} T_{a+n}^{n}\{f(x)\}= & (1-4 x)^{-1 / 2}\left[\frac{2}{1+(1-4 x)^{1 / 2}}\right]^{a-1} \\
& \cdot f\left[\frac{2 x}{1+(1-4 x)^{1 / 2}}\right]
\end{aligned}
$$

Putting $a=\lambda$ and

$$
f(x)=F_{D}^{(r)}\left[\left(\lambda, m_{i}\right):\left(\beta_{i}, \varphi_{i}\right) ;\left(\gamma, \psi_{i}\right) ; x_{1} t^{m_{1}}, \ldots, x_{r} t^{m_{r}}\right]
$$

in (3.4) and assuming that $T_{k}$-operates on $t$ alone, we get

$$
\begin{aligned}
& \sum_{n=0}^{\infty} \frac{(\lambda)_{2 n} t^{n}}{n !(\lambda)_{n}} F_{2: 0,0, \ldots, 0}^{2: 1, \ldots, 1}\left[\begin{array}{l}
\left.\left(\lambda, m_{i}\right),\left(-n, m_{i}\right):\left(\beta_{i}, \varphi_{i}\right) ; x_{1}, \ldots, x_{r}\right] \\
\left(\gamma, \psi_{i}\right),\left(1-\lambda-2 n, m_{i}\right)
\end{array}\right] \\
&=(1-4 t)^{-1 / 2}\left[\frac{2}{1+(1-4 t)^{1 / 2}}\right]^{\lambda-1} \\
& \cdot F_{D}^{(r)}\left[\left(\lambda, m_{i}\right):\left(\beta_{i}, \varphi_{i}\right) ;\left(\gamma, \psi_{i}\right) ;\right. \\
&\left.x_{1}\left(\frac{2 t}{1+(1-4 t)^{1 / 2}}\right)^{m_{1}}, \ldots, x_{r}\left(\frac{2 t}{1+(1-4 t)^{1 / 2}}\right)^{m_{r}}\right]
\end{aligned}
$$

Putting $\lambda=\gamma$ and $\psi_{i}=m_{i}$ in (3.5) we get the following generating relation for 
the Lauricella function

$$
\begin{aligned}
\sum_{n=0}^{\infty} & \frac{(\lambda)_{2 n} t^{n}}{n !(\lambda)_{n}} F_{D}^{(r)}\left[\left(-n, m_{i}\right):\left(\beta_{i}, \varphi_{i}\right) ;\left(1-\lambda-2 n, m_{i}\right) ; x_{1}, \ldots, x_{r}\right] \\
= & (1-4 t)^{1 / 2}\left[\frac{2}{1+(1-4 t)^{1 / 2}}\right]^{\lambda-1} \\
& \cdot F_{D}^{(r)}\left[\left(\lambda, m_{i}\right):\left(\beta_{i}, \varphi_{i}\right) ;\left(\lambda, m_{i}\right) ; x_{1}\left(\frac{2 t}{1+(1-4 t)^{1 / 2}}\right)^{m_{1}},\right. \\
& \left.\ldots, x_{r}\left(\frac{2}{1+(1-4 t)^{1 / 2}}\right)^{m_{r}}\right]
\end{aligned}
$$

Again by making use of the Mittal [6] operational formula

$$
\sum_{n=0}^{\infty} \frac{1}{n !} T_{a-n}^{n}\{f(x)\}=(1+x)^{a-1} f[x(1+x)]
$$

Proceeding as above we get the generating relation

$$
\begin{aligned}
& \sum_{n=0}^{\infty}(-1)^{n} \frac{(1-\lambda)_{n}}{n !}(t)^{n} F_{2: 0, \ldots, 0}^{3: 1, \ldots, 1} {\left[\begin{array}{c}
\left(\lambda, m_{i}\right),\left(\lambda, m_{i}\right)\left(-n, m_{i}\right) ; \\
\left(\beta_{i}, \varphi_{i}\right) ; x_{1}, \ldots, x_{r} \\
\left(\gamma, \psi_{i}\right),\left(\lambda-n, m_{i}\right)
\end{array}\right] } \\
&=(1+t)^{\lambda-1} F_{D}^{(r)}\left[\left(\lambda, m_{i}\right):\left(\beta_{i}, \varphi_{i}\right) ;\left(\gamma, \psi_{i}\right) ;\right. \\
&\left.x_{1}\{-t(1+t)\}^{m_{1}}, \ldots, x_{r}\{-t(1+t)\}^{m_{r}}\right]
\end{aligned}
$$

Next using the Mittal [6] operational formula,

$$
\begin{aligned}
& \sum_{n=0}^{\infty} \frac{1}{n !} T_{a-2 n}^{n}\{f(x)\}=(1+4 x)^{-1 / 2} \\
& \cdot\left[\frac{2}{1+(1+4 x)^{1 / 2}}\right]^{-a} f\left[\frac{x\left(1+(1+4 x)^{1 / 2}\right)}{2}\right] .
\end{aligned}
$$

Putting $a=\lambda$, and

$$
f(x) \equiv F_{D}^{(r)}\left[\left(\lambda, m_{i}\right):\left(\beta_{i}, \varphi_{i}\right) ;\left(\gamma, \psi_{i}\right) ; x_{1}\left(\frac{9}{8} t\right)^{m_{1}}, \ldots, x_{r}\left(\frac{9}{8} t\right)^{m_{r}}\right]
$$


in (3.9) and assuming that $T_{k}$-operates on $t$ alone we get

$$
\begin{aligned}
& \sum_{n=0}^{\infty}(-)^{n} \frac{(1-\lambda)_{2 n}}{(1-\lambda)_{n}} \frac{t^{n}}{n !} \\
& \cdot F_{4: 0, \ldots, 0,1}^{4: 1, \ldots}\left[\begin{array}{c}
\left(-n, m_{i}\right),\left(\lambda, m_{i}\right)\left(\frac{\lambda-n+1}{2}, m_{i}\right)\left(\frac{\lambda^{-n}}{2}, m_{i}\right) ; \\
\left(\beta_{i}, \varphi_{i}\right) ; x_{1}, \ldots, x_{r} \\
\left(\gamma, \psi_{i}\right),\left(\frac{\lambda-2 n}{3}, m_{i}\right),\left(\frac{\lambda-2 n+1}{3}, m_{i}\right), \\
\left(\frac{\lambda-2 n+2}{3}, m_{i}\right)
\end{array}\right] \\
& =(1+4 t)^{-1 / 2}\left[\frac{2}{1+(1+4 t)^{1 / 2}}\right]^{-\lambda} \\
& \cdot F_{D}^{(r)}\left[\left(\lambda, m_{i}\right):\left(\beta_{i}, \varphi_{i}\right) ;\left(\gamma, \psi_{i}\right) ; x_{1}\left[\frac{9}{8} t\left\{1+(1+4 t)^{1 / 2}\right\}\right]^{m_{1}}\right. \text {, } \\
& \left.\ldots, x_{r}\left[\frac{9}{8} t\left\{1+(1+4 t)^{1 / 2}\right\}\right]^{m_{r}}\right] \text {. }
\end{aligned}
$$

Putting $a=\lambda-1$ and

$$
\begin{aligned}
f(x) \equiv F^{(r)}\left[\left(\lambda, m_{i}\right):\left(\beta_{i}, \varphi_{i}\right)\right. & ;\left(\gamma, \psi_{i}\right) ; \\
& \left.x_{1}\left\{\frac{(2-m)^{2-m}}{(1-m)^{1-m}} t\right\}^{m_{1}}, \ldots, x_{r}\left\{\frac{(2-m)^{2-m}}{(1-m)^{1-m}} t\right\}\right\}^{m_{r}} \mid
\end{aligned}
$$

in (2.1) and operating $T_{k}$ on $t$ alone, we get the general result

$$
\begin{aligned}
\sum_{n=0}^{\infty} \frac{(-1)^{n} t^{n} z^{n}(\lambda)_{m n}}{n !(\lambda)_{(m-1) n}} & \sum_{k_{1}, \ldots, k_{r}=0}^{\infty} \frac{(\lambda)_{\Sigma m_{i} k_{i}}\left(\beta_{i}\right)_{k_{1} \varphi_{1}} \cdots\left(\beta_{r}\right)_{k_{r} \varphi_{r}}(-n)_{\Sigma m_{i} k_{i}}}{(\gamma)_{k_{1} \psi_{1}+\cdots+k_{r} \psi_{r}} k_{1} ! \cdots k_{r} !} \\
& \quad \frac{z^{-\Sigma m_{i} k_{i}}\left(\frac{\lambda+m n}{1-m}\right)_{\Sigma m_{i} k_{i}} \cdots\left(\frac{\lambda+m n-m}{1-m}\right)_{\Sigma m_{i} k_{i}} x_{1}^{k_{1}} \cdots x_{r}^{k_{r}}}{\left(\frac{\lambda+(m-1) n}{2-m}\right)_{\Sigma m_{i} k_{i}} \cdots\left(\frac{\lambda+(m-1) n+1-m}{2-m}\right)_{\Sigma m_{i} k_{i}}}
\end{aligned}
$$




$$
\begin{gathered}
=\frac{(1+v)^{\lambda}}{1-(m-1) v} F_{D}^{(r)}\left[\left(\lambda, m_{i}\right) ;\left(\beta_{i}, \varphi_{i}\right) ;\left(\gamma, \psi_{i}\right) ;\right. \\
x_{1}\left\{\frac{(2-m)^{2-m_{t}(1+v)}}{(1-m)^{1-m}}\right\}^{m_{1}}, \\
\left.\ldots, x_{r}\left\{\frac{(2-m)^{2-m_{t}(1+v)}}{(1-m)^{1-m}}\right\}^{m_{r}}\right]
\end{gathered}
$$

where $v=t z(1+v)^{m}$. Putting $z=1$ in (3.11) we get

$$
\begin{aligned}
& \sum_{n=0}^{\infty}(-1)^{n} \frac{(\lambda)_{m n} t^{n}}{(\lambda)_{(m-1) n} n !} \\
& \cdot_{m: 0, \ldots, 0,0}^{m: 1, \ldots}\left[\begin{array}{l}
\left.\left(\lambda, m_{i}\right),\left(\frac{\lambda+m n}{1-m}, m_{i}\right) \cdots\left(\frac{\lambda+m n-m}{1-m}, m_{i}\right) ;\left(\beta_{i}, \varphi_{i}\right) ; x_{1} \cdots x_{r}\right] \\
\left.\left(\gamma, \psi_{i}\right),\left(\frac{\lambda+(m-1) n}{2-m}, m_{i}\right) \cdots\left(\frac{\lambda+(m-1) n+1-m}{2-m}, m_{i}\right)\right]
\end{array}\right. \\
& =\frac{(1+v)^{\lambda}}{1-(m-1) v} F_{D}^{r}\left[\begin{array}{c}
\left(\lambda, m_{i}\right):\left(\beta_{i}, \varphi_{i}\right) ;\left(\gamma, \psi_{i}\right) ; \\
x_{1}\left\{\frac{(2-m)^{2-m}}{(1-m)^{1-m}} t(1+v)\right\}^{m_{1}}, \ldots, x_{r}\left\{\frac{(2-m)^{2-m}}{\left.\left.(1-m)^{1-m} t(1+v)\right\}^{m_{r}}\right]}\right]
\end{array}\right.
\end{aligned}
$$

where $v=t(1+v)^{m}$.

\section{Agarwal [8] defined $G$-function as follows}

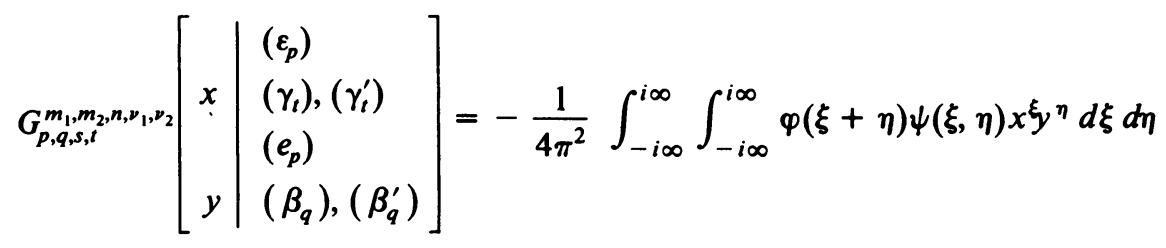


where

$$
\begin{aligned}
\psi(\xi, \eta)= & \begin{array}{c}
\prod_{j=1}^{m_{1}} \Gamma\left(\beta_{j}-\xi\right) \Pi_{j=1}^{\nu_{1}} \Gamma\left(\gamma_{j}+\xi\right) \\
\prod_{j=m_{1}+1}^{q} \Gamma\left(1-\beta_{j=1}+\xi\right) \Pi_{j=\nu_{1}+1}^{t} \Gamma\left(1-\beta_{j}^{\prime}-\xi\right) \\
\cdot \prod_{j=m_{2}+1}^{q} \Gamma\left(1-\beta_{j}^{\prime}+\eta\right) \Pi_{j=\nu_{2}+1}^{t} \Gamma\left(1-\gamma_{j}^{\prime}+\eta\right)
\end{array} \\
\varphi(\xi+\eta)= & \frac{\Pi_{j=1}^{n}\left(1-\varepsilon_{j}+\xi+\eta\right)}{\prod_{j=n+1}^{p} \Gamma\left(\varepsilon_{j}-\xi-\eta\right) \Pi_{j=1}^{s}\left(e_{j}+\xi+\eta\right)}
\end{aligned}
$$

and $0 \leqslant m_{1}<q, 0 \leqslant m_{2} \leqslant q, 0 \leqslant \nu_{1} \leqslant t, 0 \leqslant \nu_{2} \leqslant t, 0 \leqslant n<p$. Putting $a=\alpha, m=\beta+1$ and

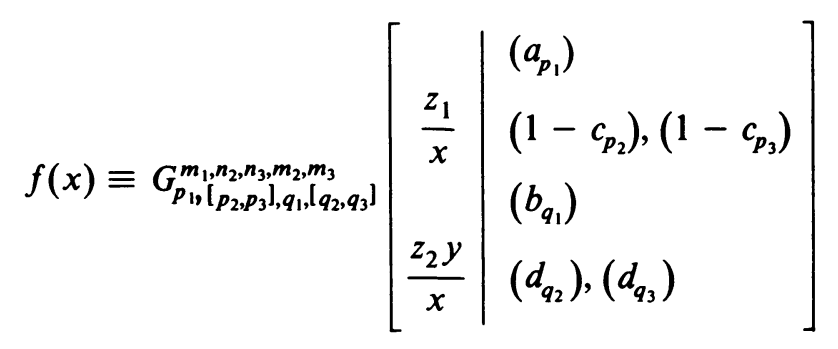

in (2.1), we get on substituting $z_{1}=x^{2}, z_{2}=x$ and $z=t / x$ after operation

$$
\begin{aligned}
& \sum_{n=0}^{\infty} \frac{(-1)^{n} t^{n}}{n !} G_{p_{1}+1,\left[p_{2}, p_{3}, q_{1}, l q_{2}, q_{3}\right]}^{m_{1}+1, n_{2}, n_{3}, m_{2}, m_{3}}\left[\begin{array}{l|l}
\left(a_{p_{1}}\right), \alpha-n \beta \\
\left(1-c_{p_{2}}\right),\left(1-c_{p_{3}}\right) \\
\left(b_{q_{1}}\right), \alpha-n-n \beta \\
\left(d_{q_{2}}\right),\left(d_{q_{3}}\right)
\end{array}\right] \\
& =\frac{(1+v)^{1-\alpha}}{(1-\beta v)} G_{p_{1}, I_{p_{2}, p_{3}, q_{1},\left[q_{2}, q_{3}\right]}^{m_{1}, n_{2}, n_{3}, m_{2}, m_{3}}}\left[\begin{array}{l|l}
\frac{x}{1+v} & \begin{array}{l}
\left(a_{p_{1}}\right) \\
\left(1-c_{p_{2}}\right),\left(1-c_{p_{3}}\right) \\
\left(b_{q_{1}}\right) \\
\frac{y}{1+v}
\end{array}
\end{array}\right]
\end{aligned}
$$

where $v=t(1+v)^{\beta+1}$, which is due to Sharma and Abiodun [1].

ACKNowledgement. I am thankful to Dr. R. P. Agarwal for guidance during the preparation of the paper.

\section{REFERENCES}

1. B. L. Sharma and R. F. Abiodun, Generating function for generalized function of two variables, Proc. Amer. Math. Soc. 46 (1974), 69-72.

2. H. M. Srivastava, A generating function for certain coefficients involving several complex variables, Proc. Nat. Acad. Sci. U. S. A. 67 (1970), 1079-1080. 
3. A new class of generating functions involving several complex variables, Nederl. Akad. Wetensch. Proc. Ser. A. 74 = Indag. Math. 33 (1971), 483-486.

4. H. M. Srivastava and L. Carlitz, Some hypergeometric polynomials associated with Lauricella function of several variables. I, Mat. Vestnik 13 (28) (1976), 41-47.

5. H. M. Srivastava and M. C. Dauost, On Eulerian integrals associated with Kampé de Fériet's function, Publ. Inst. Math. 9 (23) (1969), 199-202.

6. H. B. Mittal, Some generating functions, Univ. Lisboa Revista Fac. Ci. A (2) 13 (1969/70), 43-54.

7. 73-84. , Polynomials defined by generating relations, Trans. Amer. Math. Soc. 168 (1972),

8. R. P. Agarwal, An extension of Meijer's G-function, Proc. Nat. Acad. Sci. India Part A 31 (1965), 536-546.

DepartMent OF MATHEMATICS AND ASTRONOMY, LUCKNOW UNIVERSITY, LUCKNOW, INDIA 